\title{
ON HARDY'S INEQUALITY AND ITS GENERALIZATION
}

\author{
Masako Izumi, Shin-ichi Izumi and Gordon M. Petersen
}

(Received March 5, 1969)

1. G. H. Hardy, [1], p. 239 has proved the following

THEOREM A. If $p>1, a_{n} \geqq 0,(n=1,2, \cdots)$ and $A_{n}=a_{1}+a_{2}+\cdots$ $+a_{n}$, then

$$
\sum_{n=1}^{\infty}\left(A_{n} / n\right)^{p}<\left(\frac{p}{p-1}\right)^{p} \sum_{n=1}^{\infty} a_{n}^{p}
$$

unless all the $a_{n}$ vanish. The constant $(p /(p-1))^{p}$ is best possible.

In [2], pp. 273-275, he proved that the arithmetic mean of $\left(a_{n}\right)$ in (1) can be replaced by a more general mean which contains the Euler mean, Cesàro mean and Hölder mean as particular cases. Another more general case has been studied in [3] and [4] where the following has been proved.

THEOREM B. Let $C=\left(c_{m, k}\right)$ be a positive triangular matrix (i.e. $c_{m, k}=0$ for $k>m$, and $c_{m, k}>0$ for $\left.k \leqq m(m=1,2, \cdots)\right)$, satisfying

$$
0<{ }_{c_{m, k}}^{c_{n, k}} \leqq A_{1} \quad \text { for all } k \leqq m \leqq n,
$$

and there exists a sequence $f(k), f(k) \nearrow \infty$ such that

$$
0<f(n) c_{n, k} / f(m) c_{m, k} \leqq A_{2}, \quad \text { for all } k \leqq m \leqq n
$$

and

$$
\sum_{n=k}^{\infty}[f(n)]^{-p} \leqq A_{3}[f(k)]^{1-p}, \quad p>1
$$

where $A_{1}, A_{2}$ and $A_{3}$ are constants.

If $a_{n} \geqq 0$, and if $p>1$ 
602 M. IZUMI. S. IZUMI AND G. M. PETERSEN

$$
\sum_{m=1}^{\infty}\left(\sum_{k=1}^{m} c_{m, k} a_{k}\right)^{p} \leqq A \sum_{m=1}^{\infty}\left(a_{m} f(m) c_{m, m}\right)^{p}
$$

The case $c_{m, k}=1 / m, k \leqq m, c_{m, k}=0, k>m$, and $f(k)=k$ satisfies the conditions of Theorem $\mathrm{B}$ and (5) reduces to (1).

2. In this paper we shall investigate various generalizations of these two theorems, the letters $A, A_{1}, A_{2}$ etc. will denote various constants independent of the terms under the summation sign.

THEOREM 1. Let $p>1, a_{m} \geqq 0(m=1,2, \cdots), g(m)>0(m=1,2, \cdots)$ and $C=\left(c_{n, k}\right)$ be a positive triangular matrix. If

$$
\sum_{m=1}^{\infty} c_{m, m}<\infty
$$

and

$$
\sum_{m=n}^{\infty} g(m) c_{m, n}^{p} \leqq A_{1} g(n) c_{n, n}^{p-1} \quad \text { for all } n \geqq 1
$$

then

$$
\sum_{m=1}^{\infty} g(m)\left(\sum_{n=1}^{m} c_{m, n} a_{n}\right)^{p} \leqq A_{2} \sum_{m=1}^{\infty} g(m) a_{m}^{\nu}
$$

Proof. Denote by $U$ and $V$ the left hand side and right hand side sums, respectively, in (8). Using Minkowski's inequality and (7)

$$
U^{1 / p} \leqq \sum_{n=1}^{\infty} a_{n}\left(\sum_{m=n}^{\infty} g(m) c_{m, n}^{p}\right)^{1 / p} \leqq A_{1}^{1 / p} \sum_{n=1}^{\infty} a_{n}[g(n)]^{1 / p} c_{n, n}^{(p-1) / p}
$$

By Hölder's inequality and (6)

$$
U^{1 / p} \leqq A_{2}\left(\sum_{n=1}^{\infty} a_{n}^{p} g(n)\right)^{1 / p}\left(\sum_{n=1}^{\infty} c_{n, n}\right)^{1 / q} \leqq A V^{1 / p}
$$

where $1 / p+1 / q=1$. This completes the proof of the Theorem 1 .

As an immediate consequence we have

COROLLARY 1.1. If (6) and 


$$
g(m) c_{m, n}^{p} \leqq A g(n) c_{n, n}^{p-1} c_{m, m} .
$$

for all $m \geqq n \geqq 1$, hold, then (8) must follow.

Let $g(m)=1(m=1,2, \cdots)$, in Theorem 1 to obtain

COROLlary 1.2. If (6) and

$$
\sum_{m=n}^{\infty} c_{m, n}^{p} \leqq A c_{n, n}^{p-1} \quad \text { for all } n \geqq 1
$$

then

$$
\sum_{m=1}^{\infty}\left(\sum_{n=1}^{m} c_{m, n} a_{n}\right)^{p} \leqq A \sum_{m=1}^{\infty} a_{m}^{p}
$$

Condition (6) is quite restrictive and we shall now attempt to find a more satisfactory alternative.

THEOREM 2. Let $a_{m} \geqq 0(m=1,2, \cdots), g(m)>0(m=1,2, \cdots)$ and $C=\left(c_{n, k}\right)$ be a positive triangular matrix which satisfies the conditions

$$
\sum_{i=\nu}^{\infty} g(m) c_{m, \mu} c_{m, \nu} \leqq A_{1} g(\nu) c_{\nu, \mu} \quad \text { for all } \mu \leqq \nu
$$

then

$$
\sum_{m=1}^{\infty} g(m)\left(\sum_{\nu=1}^{m} c_{m, \nu} a_{\nu}\right)^{2} \leqq A_{2} \sum_{m=1}^{\infty} g(m) a_{m}^{2} .
$$

ProOF. From the left hand side of (12)

$$
\begin{aligned}
\sum_{m=1}^{\infty} g^{\prime}(m)\left(\sum_{\nu=1}^{m} c_{m, \nu} a_{\nu}\right)^{2} & =\sum_{m=1}^{\infty} g(m)\left(\sum_{\nu=1}^{m} c_{m, \nu} a_{\nu}\right)\left(\sum_{\mu=1}^{m} c_{m, \mu} a_{\mu}\right) \\
& =\sum_{\nu=1}^{\infty} a_{\nu}\left(\sum_{m=\nu}^{\infty} c_{m, \nu} g(m)\left(\sum_{\mu=1}^{m} c_{m, \mu} a_{\mu}\right)\right) \\
& \leqq \sum_{\nu=1}^{\infty} a_{\nu} \sum_{m=\nu}^{\infty} c_{m, \nu} g(m)\left(\sum_{\mu=1}^{\nu} c_{m, \mu} a_{\mu}+\sum_{\mu=\nu}^{m} c_{m, \mu} a_{\mu}\right) \\
& =\sum_{\nu=1}^{\infty} a_{\nu} \sum_{\mu=1}^{\nu} a_{\mu} \sum_{m=\nu}^{\infty} c_{m, \mu} c_{m, \nu} g(m)+\sum_{\nu=1}^{\infty} a_{\nu} \sum_{\mu=\nu}^{\infty} a_{\mu} \sum_{m=\mu}^{\infty} c_{m, \mu} c_{m, \nu} g(m) \\
& =S+T .
\end{aligned}
$$


604 M. IZUMI， S. IZUMI AND G. M. PETERSEN

From condition (11) and Schwarz's inequality, it follows that

$$
\begin{aligned}
S & \leqq A_{3} \sum_{\nu=1}^{\infty} a_{\nu} \sum_{\mu=1}^{\nu} a_{\mu} g(\nu) c_{\nu, \mu}=A_{3} \sum_{\nu=1}^{\infty} a_{\nu}(g(\nu))^{\frac{1}{2}}(g(\nu))^{\frac{1}{2}} \sum_{\mu=1}^{\nu} a_{\mu} c_{\nu, \mu} \\
& \leqq A_{3}\left(\sum_{\nu=1}^{\infty} g(\nu) a_{\nu}^{2}\right)^{1 / 2}\left(\sum_{\nu=1}^{\infty} g(\nu)\left(\sum_{\mu=1}^{\nu} c_{\nu, \mu} a_{\mu}\right)^{2}\right)^{1 / 2}=A_{3} S^{\prime}
\end{aligned}
$$

and

$$
T \leqq A_{4} \sum_{\nu=1}^{\infty} a_{\nu} \sum_{\mu=\nu}^{\infty} a_{\mu} g(\mu) c_{\mu, \nu}=A_{4} \sum_{\mu=1}^{\infty} a_{\mu} g(\mu) \sum_{\nu=1}^{\prime \prime} c_{\mu, \nu} a_{\nu} \leqq A_{4} S^{\prime}
$$

Combining (13) and (14), we have the desired inequality (12).

An immediate consequence of Theorem 2 is the following

COROllary 2.1. Let $a_{m} \geqq 0(m=1,2, \cdots), g(m)>0(m=1,2, \cdots)$, $C=\left(c_{m, k}\right)$ be a positive triangular matrix and $\alpha$ any real number. If

$$
\sum_{m=\nu}^{\infty}\left(c_{m, m}^{1+\alpha} / g(m)^{\alpha}\right) \leqq A c_{\nu, \nu}^{\alpha} / g(\nu)^{\alpha} \quad \text { for all } \nu \geqq 1
$$

and

$$
(g(m))^{(1+\alpha) / 2} c_{n, n}^{(1+\alpha) / 2} c_{m, \mu} \leqq A_{1}(g(n))^{(1+\alpha) / 2} c_{m, m}^{(1+\alpha) / 2} c_{n, \mu}
$$

for all $m \geqq n \geqq \mu$, then inequality (12) holds.

The case $\alpha=0$ gives

COROLlary 2.2. If $\sum_{m=1}^{\infty} c_{m, m}<\infty$ and

$$
g(m) c_{n, n} c_{m, \mu}^{2} \leqq A g(n) c_{m, m} c_{n, \mu}^{2}
$$

for all $m \geqq n \geqq \mu$, then inequality (12) holds.

Another corollary of Theorem 2 is

Corollary 2.3. Let $a_{m} \geqq 0(m=1,2, \cdots), g(m)>0(m=1,2, \cdots)$, $C=\left(c_{m, k}\right)$ be a positive triangular matrix and $\beta$ any real number. If 


$$
\sum_{m=\nu}^{\infty}\left(c_{m, \nu} c_{m, m}^{1+\beta}(g(m))^{-\beta}\right) \leqq A c_{\nu, \nu}^{1+\beta}(g(\nu))^{-\beta} \quad \text { for all } \nu \geqq 1
$$

and

$$
g(m)^{1+\beta} c_{n, n}^{1+\beta} c_{m, \mu} \leqq A_{1} g(n)^{1+\beta} c_{m, m}^{1+\beta} c_{n, \mu} \quad \text { for all } m \geqq n \geqq \mu,
$$

then inequality (12) holds.

For the case $\beta=0$, we have

COROLLARY 2.4. If

$$
\sum_{m=\nu}^{\infty} c_{m, \nu} c_{m, m}<A c_{\nu, \nu} \quad \text { for all } \nu \geqq 1
$$

and

$$
g(m) c_{n, n} c_{m, \mu} \leqq A_{1} g(n) c_{m, m} c_{n, \mu} \quad \text { for all } m \geqq n \geqq \mu,
$$

then inequality (12) holds.

In Theorem 2, let us consider the case $c_{m, k}=p_{k} / q_{m},\left(p_{k}>0, q_{m}>0\right)$ and $g(m)=1$. We have

COROLLARY 2.5. If $\sum_{m=\nu}^{\infty} q_{m}^{-2} \leqq A\left(p_{\nu} q_{\nu}\right)^{-1}$ then

$$
\sum_{m=1}^{\infty}\left(\frac{1}{q_{m}} \sum_{\nu=1}^{m} p_{\nu} a_{\nu}\right)^{2} \leqq A_{1} \sum_{\nu=1}^{\infty} a_{\nu}^{2} .
$$

By the same substitution in Theorem 1 we obtain

COROLLARY 2.6. If $\sum_{m=1}^{\infty}\left(p_{m} / q_{m}\right)<\infty$ and

$$
\sum_{m=\nu}^{\infty} q_{m}^{-p} \leqq A\left(p_{\nu}^{-1} q_{\nu}^{-p+1}\right) \quad \text { for all } \nu \geqq 1
$$

then

$$
\sum_{m=1}^{\infty}\left(\frac{1}{q_{m}} \sum_{\nu=1}^{m} p_{\nu} a_{\nu}\right)^{p} \leqq A_{1} \sum_{m=1}^{\infty} a_{m}^{p} .
$$


3. By an $M$ matrix we shall denote a positive triangular matrix $C=\left(c_{m, k}\right)$ which satisfies

$$
0 \leqq \frac{c_{m, k}}{c_{n, k}} \leqq K \quad(0 \leqq k \leqq n \leqq m)
$$

and for which there exists an $f(m) \nearrow \infty$ such that

$$
\frac{c_{m, k}}{c_{n, k}} \leqq K_{1} \frac{f(n)}{f(m)} \quad(0 \leqq k \leqq n \leqq m)
$$

where $K$ and $K_{1}$ are constants.

THEOREM 3. Let $p>1$. If $C=\left(c_{m, k}\right)$ is an $M$ matrix and $a_{k} \geqq 0$ $(k=1,2, \cdots)$, and if

$$
\sum_{m=1}^{\infty} \frac{c_{m, m}}{(f(m))^{p-1}} \quad \text { converges }
$$

and

$$
\sum_{m=\nu}^{\infty} \frac{c_{m, m}}{(f(m))^{p-1}} \leqq \frac{A}{(f(\nu))^{p-1}}
$$

it follow that

$$
\sum_{m=1}^{\infty} c_{m, m} f(m)\left\{\sum_{\nu=1}^{m} c_{m, \nu} a_{\nu}\right\}^{\prime \prime} \leqq A_{1} \sum_{m=1}^{\infty} c_{m, m} f(m) a_{m}^{p} .
$$

Proof. We first observe that from Lemma 1 in [4] we have

$$
\begin{aligned}
\left(\sum_{\nu=1}^{m} c_{m, \nu} a_{\nu}\right)^{p} & \leqq A_{2} \sum_{\nu=1}^{m} c_{m, \nu} a_{\nu}\left(\sum_{r=1}^{\nu} c_{m, r} a_{r}\right)^{p-1} \\
& \leqq A_{3} \sum_{\nu=1}^{m} c_{m, \nu} a_{\nu}\left(\frac{f(\nu)}{f(m)}\right)^{\nu-1}\left(\sum_{r=1}^{\nu} c_{\nu, r} a_{r}\right)^{p-1}
\end{aligned}
$$

Hence

$$
\begin{aligned}
S_{N} & =\sum_{m=1}^{N} c_{m, m} f(m)\left\{\sum_{\nu=1}^{m} c_{m, \nu} a_{\nu}\right\}^{p} \\
& \leqq A_{3} \sum_{m=1}^{N}\left[\sum_{\nu=1}^{m} c_{m, m} f(m) c_{m, \nu} a_{\nu}\left(\frac{f(\nu)}{f(m)}\right)^{p-1}\left(\sum_{r=1}^{\nu} c_{\nu, r} a_{r}\right)^{p-1}\right]
\end{aligned}
$$


and it follows that

$$
S_{N} \leqq A_{3} \sum_{\nu=1}^{N}\left(\sum_{r=1}^{\nu} c_{\nu, r} a_{r}\right)^{p-1}\left[\sum_{m=\nu}^{N} c_{m, m} f(m) c_{m, \nu} a_{\nu}\left(\frac{f(\boldsymbol{\nu})}{f(m)}\right)^{p-1}\right]
$$

Also, we have by applying (16) and then (17),

$$
\begin{aligned}
& \sum_{m=\nu}^{N} c_{m, m} f(m) c_{m, \nu} a_{\nu}\left(\frac{f(\boldsymbol{\nu})}{f(m)}\right)^{p-1}=a_{\nu}(f(\boldsymbol{\nu}))^{p-1} \sum_{m=\nu}^{N} c_{m, m} c_{m, \nu}(f(m))^{2-p} \\
& \quad=a_{\nu}(f(\boldsymbol{\nu}))^{p-1} c_{\nu, \nu} \sum_{m=\nu}^{N} c_{m, m} \frac{c_{m, \nu}}{c_{\nu, \nu}}(f(m))^{2-p} \\
& \quad \leqq a_{\nu}(f(\boldsymbol{\nu}))^{p-1} c_{\nu, \nu} K_{1} \sum_{m=\nu}^{N} c_{m, m} f(\nu)(f(m))^{1-p} \\
& \leqq a_{\nu}(f(\boldsymbol{\nu}))^{p} c_{\nu, \nu} K_{1} \sum_{m=\nu}^{N} c_{m, m}(f(m))^{1-p} \leqq a_{\nu} f(\nu) c_{\nu, \nu} K_{1} A \leqq A_{4} a_{\nu} f(\nu) c_{\nu, \nu}
\end{aligned}
$$

Substituting this relation in the previous expression and using Hölder's inequality

$$
\begin{gathered}
\sum_{m=1}^{N} c_{m, m} f(m)\left(\sum_{\nu=1}^{m} c_{m, \nu} a_{\nu}\right)^{p} \leqq A_{3} A_{4} \sum_{m=1}^{N}\left(\sum_{\nu=1}^{m} c_{m, \nu} a_{\nu}\right)^{p-1} a_{m} f(m) c_{m, m} \\
\leqq A_{5}\left\{\sum_{m=1}^{N} c_{m, m} f(m) a_{m}^{p}\right\}^{1 / p}\left\{\sum_{m=1}^{N} c_{m, m} f(m)\left(\sum_{\nu=1}^{m} c_{m, \nu} a_{\nu}\right)^{p}\right\}^{1 / q},
\end{gathered}
$$

where $1 / p+1 / q=1$.

Dividing both sides of the inequality by the last factor on the right and raising to the $p$ th power,

$$
\sum_{m=1}^{N} c_{m, m} f(m)\left(\sum_{\nu=1}^{m} c_{m, \nu} a_{\nu}\right)^{p} \leqq A_{1} \sum_{m=1}^{\infty} c_{m, m} f(m) a_{m}^{p} .
$$

By letting $N$ tend to infinity, we prove our theorem.

Suppose an additional condition is satisfied by the matrix, namely

$$
c_{n, n,} f(m) \nearrow
$$

then let

$$
a_{n}=\left(c_{n, n} f(n)\right)^{-1 / p} \mu_{n}
$$


608 M. IZUMI, S. IZUMI AND G. M. PETERSEN

and substituting in (19), we have

$$
\sum_{m=1}^{\infty}\left(\sum_{\nu=1}^{m} c_{m, \nu} \mu_{\nu}\right)^{p} \leqq A_{1} \sum_{m=1}^{\infty} \mu_{m}^{p} .
$$

A matrix satisfying (15), (16), (18) and (20) in the case $p=2$ is given by

$$
c_{m, \nu}= \begin{cases}\boldsymbol{\nu}^{1 / 2} m^{-3 / 2} & (\boldsymbol{\nu} \leqq m) \\ 0 & (\boldsymbol{\nu}>m) .\end{cases}
$$

For this matrix

$$
\frac{c_{m, \nu}}{c_{n, \nu}}=\frac{\nu^{1 / 2} m^{-3 / 2}}{\nu^{1 / 2} n^{-3 / 2}} \leqq 1 \quad(0 \leqq \nu \leqq n \leqq m)
$$

if $f(m)=m^{3 / 2}$,

$$
\frac{c_{m, \nu}}{c_{n, \nu}} \leqq \frac{f(n)}{f(m)} \quad(0 \leqq \nu \leqq n \leqq m)
$$

Furthermore,

$$
\sum_{m=\nu}^{\infty} c_{m, m}[f(m)]^{-1}=\sum_{m=\nu}^{\infty} m^{-1} m^{-3 / 2} \leqq \int_{\nu-1}^{\infty} x^{-5 / 2} d x \leqq A \nu^{-3 / 2}=\frac{A}{f(\nu)},
$$

and $c_{m, m} f(m)=m^{1 / 2} \nearrow$ so that the stated conditions are satisfied. From (21) this implies

$$
\sum_{m=1}^{\infty}\left(\sum_{\nu=1}^{m} \nu^{1 / 2} m^{-3 / 2} \mu_{\nu}\right)^{2} \leqq A_{1} \sum_{m=1}^{\infty} \mu_{m}^{2} .
$$

4. We next turn our attention to

THEOREM 4. If $a_{n} \geqq 0(n=1,2, \cdots)$, and $C=\left(c_{m, k}\right)$ is a positive triangular matrix, satisfying

$$
\sum_{m=n}^{\infty}\left(c_{m, k}\right)^{p} \leqq A_{1} n^{-p+1} \quad \text { for } 1 \leqq k \leqq n
$$

$(n=1,2, \cdots)$ for some $p>1$, then

$$
\sum_{n=1}^{\infty}\left(\sum_{k=1}^{n} c_{n, k} a_{k}\right)^{p} \leqq A_{2} \sum_{n=1}^{\infty} a_{n}^{p} .
$$


We shall also prove

THEOREM 5. If $p>1(n=1,2, \cdots), f(m) \geqq 0(m=1,2, \cdots)$ and $C=\left(c_{m, k}\right)$ is a positive triangular matrix, satisfying the condition

$$
\sum_{n=k}^{\infty} n^{\epsilon}\left(c_{n, k}\right)^{p} \leqq A_{1} k^{\varepsilon}\left(f(k) c_{k, k}\right)^{p}
$$

for a small $\varepsilon>0$ and for all $k \geqq 1$, then

$$
\sum_{n=1}^{\infty}\left(\sum_{k=1}^{n} c_{n, k} a_{k}\right)^{p} \leqq A_{2} \sum_{n=1}^{\infty} n^{-1}\left(n a_{n} f(n) c_{n, n}\right)^{p}
$$

Proof of Theorem 4. Let $1 / p+1 / q=1$ and $0<r<1 / q$. Then by Hölder's inequality,

$$
\begin{gathered}
\sum_{m=1}^{\infty}\left(\sum_{k=1}^{m} c_{m, k} a_{k}\right)^{p}=\sum_{m=1}^{\infty}\left(\sum_{k=1}^{m} k^{r} c_{m, k} a_{k} k^{-r}\right)^{p} \leqq \sum_{m=1}^{\infty}\left(\sum_{k=1}^{m} k^{r p}\left(c_{m, k} a_{k}\right)^{p}\right)\left(\sum_{k=1}^{m} k^{-r q}\right)^{p / q} \\
\leqq A_{3} \sum_{m=1}^{\infty} m^{p-r p-1} \sum_{k=1}^{m} k^{r p}\left(c_{m, k} a_{k}\right)^{p} \leqq A_{4} \sum_{k=1}^{\infty} k^{r p} a_{k}^{p} \sum_{m=k}^{\infty} m^{p-r p-1}\left(c_{m, k}\right)^{p}
\end{gathered}
$$

Let $C_{n, k}=\sum_{m=n}^{\infty}\left(c_{m, k}\right)^{p}(k \leqq n)$ so that by $(22)$

$$
C_{n, k} \leqq A_{1} n^{-p+1} \quad \text { for all } k \leqq n
$$

Hence, for the inner sum on the right hand side of (26),

$$
\begin{aligned}
\sum_{m=k}^{\infty} m^{p-r p-1}\left(c_{m, k}\right)^{p} & =\sum_{m=k}^{\infty} m^{p-r p-1}\left(C_{m, k}-C_{m+1, k}\right) \\
& =k^{p-r p-1} C_{k, k}+\sum_{m=k}^{\infty}\left[(m+1)^{p-r p-1}-m^{p-r p-1}\right] C_{m+1, k} \\
& \leqq A_{1} k^{-r p}+A_{5} \sum_{m=k}^{\infty}(m+1)^{p-r p-2}(m+1)^{-p+1} \leqq A_{6} k^{-r p}
\end{aligned}
$$

Substituting (27) into (26) we complete the proof of Theorem 4.

Proof of Theorem 5. As before, let $1 / p+1 / q=1$ and $0<r<1 / q$. In (26) let $r$ be chosen so that $\varepsilon=p-r p-1$, then (24) becomes 


$$
\sum_{m=k}^{\infty} m^{p-r p-1}\left(c_{m, k}\right)^{p} \leqq A_{1} k^{p-r p-1}\left(f(k) c_{k, k}\right)^{p}
$$

and then, by (26)

$$
\sum_{m=1}^{\infty}\left(\sum_{k=1}^{m} c_{m, k} a_{k}\right)^{p} \leqq A_{3} \sum_{k=1}^{\infty} k^{-1}\left(k a_{k} f(k) c_{k, k}\right)^{p}
$$

which is the required result.

Consider the Nörlund mean

$$
c_{n, k}=\frac{p_{n-k+1}}{P_{n}} \quad \text { for } k \leqq n \text { and } c_{n, k}=0 \text { for } k>n,
$$

where $p_{n} \geqq 0$ and $P_{n}=p_{1}+p_{2}+\cdots+p_{n}>0$. Then we have the following

COROLLARY 5.1. If $a_{n} \geqq 0, f(m) \geqq 0, p_{n} \geqq 0$ and

$$
\sum_{n=k}^{\infty} n^{\varepsilon}\left(p_{n-k+1} / P_{n}\right)^{p} \leqq A_{1} k^{\varepsilon}\left(f(k) / P_{k}\right)^{p}
$$

for an $\varepsilon>0$ and all $k \geqq 1$, then we have

$$
\sum_{n=1}^{\infty}\left(\sum_{k=1}^{n} p_{n-k} a_{k} / P_{n}\right)^{p} \leqq A_{2} \sum_{n=1}^{\infty} n^{-1}\left(n a_{n} f(n) / P_{n}\right)^{p}
$$

For example, we take $p_{n} \cong 1 / \log n$, then $P_{n} \cong n \log n$ and (28) is satisfied for $0<\varepsilon<p-1$ and $f(n)=n^{1 / p} / \log (n+1)$. Thus we have

$$
\sum_{n=1}^{\infty}\left(\frac{\log n}{n} \sum_{k=1}^{n} \frac{a_{k}}{\log (n-k+1)}\right)^{p} \leqq A_{2} \sum_{n=1}^{\infty} a_{n}^{p} .
$$

We shall next consider the case $p_{n} \cong n^{-\alpha}(0 \leqq \alpha<1)$ then $P_{n} \cong n^{1-\alpha}$ and (28) is satisfied for $0<\varepsilon<p-1$ and $[f(n)]^{p}=n^{1-\alpha p}$, $\log n$ or 1 according as $0 \leqq \alpha<1 / p, \alpha=1 / p$ or $\alpha>1 / p$. Then we have

$$
\sum_{n=1}^{\infty}\left(n^{\alpha-1} \sum_{k=1}^{n} \frac{a_{k}}{(n-k+1)^{\alpha}}\right)^{p} \leqq A_{2} \sum_{n=1}^{\infty} a_{n}^{p} g(n)
$$

where $g(n)$ is $1, \log n$ or $n^{\alpha-1}$ according as $0 \leqq \alpha<1 / p, \alpha=1 / p$ or $1 / p<\alpha<1$. 
Finally we consider the case $p_{n}=1 / n$, then $P_{n} \cong \log n$ and $f(n)=1$. The inequality that results is

$$
\sum_{n=1}^{\infty}\left(\frac{1}{\log (n+1)} \sum_{k=1}^{n} \frac{a_{k}}{n-k+1}\right)^{p} \leqq A_{2} \sum_{n=1}^{\infty} \frac{a_{n}^{p} n^{p-1}}{\log (n+1)}
$$

Another corollay is

COROLlARY 5.2. Let $p>1, f(k) \geqq 0(k=1,2, \cdots)$, and $C=\left(c_{n, k}\right) a$ positive triangular matrix. If

$$
\sum_{m=k}^{\infty} m^{s}\left(c_{m, k}\right)^{p} \leqq A_{1} k^{c}[f(k)]^{p}
$$

for an $\varepsilon>0$ and for all $k \geqq 1$, then

$$
\sum_{n=1}^{\infty}\left(\sum_{k=1}^{n} \frac{c_{n, k}}{k}\right)^{p} \leqq A_{2} \sum_{n=1}^{\infty} \frac{[f(n)]^{p}}{n} .
$$

5. In the previous paragraph, the conditions were imposed on the column sums of the matrix. We shall exhibit a theorem involving row sums.

THEOREM 6. Let $C=\left(c_{n, k}\right)$ be a positive triangular matrix and $a_{n} \geqq 0$ $(n=1,2, \cdots), f(n) \geqq 0(n=1,2, \cdots), g(n) \geqq 0(n=1,2, \cdots)$, and $p>2$. If

$$
\sum_{k=1}^{n}\left(c_{n, k}\right)^{p} \leqq A_{1}\left(f(n) c_{n, n}\right)^{p} \quad \text { for all } n \geqq 1
$$

and

$$
\sum_{n=k}^{\infty} n^{c}\left(f(n) c_{n, n}\right)^{p} \leqq A_{2} k^{\varepsilon}\left(f(k) g(k) c_{k, k}\right)^{p}
$$

for an $\varepsilon>0$ and for all $k \geqq 1$, then we have

$$
\sum_{n=1}^{\infty}\left(\sum_{k=1}^{n} c_{n, k} a_{k}\right)^{p} \leqq A_{3} \sum_{n=1}^{\infty} n^{-2}\left(n a_{n} f(n) g(n) c_{n, n}\right)^{p}
$$

PROOF. Let $p>2,1 / p+1 / q=1$ and $b>0$. By Hölder's inequality

$$
\sum_{m=1}^{\infty}\left(\sum_{k=1}^{m} c_{m, k} a_{k}\right)^{p}=\sum_{m=1}^{\infty}\left(\sum_{k=1}^{m} k^{b} a_{k} k^{-b} c_{m, k}\right)^{p}
$$


612

$$
\leqq \sum_{m=1}^{\infty}\left(\sum_{k=1}^{m} k^{b p} a_{k}^{p}\right)\left(\sum_{j=1}^{m} j^{-b q} c_{m, j}^{q}\right)^{p / q}=\sum_{k=1}^{\infty} k^{b p} a_{k}^{p} \sum_{m=k}^{\infty}\left(\sum_{j=1}^{m} j^{-b q} c_{m, j}^{q}\right)^{p / l} .
$$

Using Hölder's inequality again,

$$
\begin{aligned}
S & =\sum_{m=k}^{\infty}\left(\sum_{j=1}^{m} j^{-b p} c_{m, j}^{q}\right)^{p / q}=\sum_{m=k}^{\infty}\left(\sum_{j=1}^{m} c_{m j}^{p}\right)\left(\sum_{r=1}^{m} r^{-b p / p-2}\right)^{p-2} \\
& \leqq A_{4} \sum_{m=k}^{\infty} m^{p-b p-2} \sum_{j=1}^{m} c_{m, j}^{p}
\end{aligned}
$$

if $0<b<(p-2) / p$. We take $b$ close to $p-2 / p$ so that $\varepsilon=p-b p-2$, then (29) and (30) imply that

$$
S \leqq A k^{-b p-2}\left(k f(k) g(k) c_{k, k}\right)^{p}
$$

and

$$
\sum_{m=1}^{\infty}\left(\sum_{k=1}^{m} c_{m, k} a_{k}\right)^{p} \leqq A_{3} \sum_{k=1}^{\infty} k^{-2}\left(k a_{k} f(k) g(k) c_{k, k}\right)^{p},
$$

which completes the proof.

In the particular case $c_{n, k}=p_{n-k+1} / P_{n}$ for $k \leqq n$ and $c_{n, k}=0$ for $k>n$, $P_{n}=p_{1}+p_{2}+\cdots+p_{n}>0(n=1,2, \cdots),(29)$ is satisfied when

$$
f(n)=\left(\sum_{k=0}^{n} p_{k}^{p}\right)^{1 / p}
$$

and condition (30) is satisfied when

$$
\left(f(k) g(k) / P_{k}\right)^{p}=k^{-\varepsilon} \sum_{n=k}^{\infty} n^{\varepsilon} . f(n)^{p} P_{n}^{-p} .
$$

The conclusion of Theorem 6 becomes

$$
\sum_{n=1}^{\infty}\left(P_{n}^{-1} \sum_{k=0}^{n} p_{n-k} a_{k}\right)^{p} \leqq A \sum_{n=1}^{\infty} a_{n}^{p} n^{p-2-\varepsilon} \sum_{m=n}^{\infty} m^{\varepsilon}\left(P_{m}^{-p} \sum_{k=0}^{m} p_{k}^{p} .\right)
$$

Thus we have

COROLlARY 6.1. Let $\varepsilon>0$ and $p>1$. If $\left(p_{n}\right)$ is any positive sequence and $P_{n}=p_{1}+p_{2}+\cdots+p_{n}(n=1,2, \cdots)$, then (34) holds for any series $\sum a_{k}$ with positive terms. 


\section{REFERENCES}

[1] G. H. Hardy, J. E. Littlewood and G. Polya, Inequalities, Cambridge (1952).

[2] G. H. HARdY, Divergent series, Cambridge (1948).

[ 3 ] G. M. Peterson, An inequality of Hardy's, Quart. J. Math. Oxford Ser., (2) 13(1962), $237-240$.

[4] G. S. Davies and G. M. Peterson, On an inequality of Hardy's (II), Quart. J. Math. Oxford Ser., (2) 15(1964), 35-40.

DEPARTMENT OF MATHEMATICS

THE AUSTRALIAN NATIONAL UNIVERSITY

CANBERra, Australia 